\title{
The Critical Role of Aromatic-Aromatic Drug- Polymer Interactions to Provide Nanomedicines Containing Chloroquine: A Simple Proposal to Provide High Encapsulation, High Stability, and Prolonged Drug Release
}

\section{María Gabriela Villamizar-Sarmiento}

Universidad de Chile Facultad de Ciencias Quimicas y Farmacéuticas: Universidad de Chile Facultad de Ciencias Quimicas y Farmaceuticas

Ignacio Moreno-Villoslada

Universidad Austral de Chile

Felipe Oyarzun-Ampuero ( $\nabla$ foyarzuna@ciq.uchile.cl )

Universidad de Chile Facultad de Ciencias Quimicas y Farmacéuticas: Universidad de Chile Facultad de Ciencias Quimicas y Farmaceuticas https://orcid.org/0000-0002-4951-0701

\section{Research}

Keywords: Chloroquine, aromatic polymer, self-assembly, controlled release, drug delivery.

Posted Date: November 12th, 2020

DOI: https://doi.org/10.21203/rs.3.rs-101145/v1

License: (c) (1) This work is licensed under a Creative Commons Attribution 4.0 International License. Read Full License 


\section{Abstract}

Background: Chloroquine (CQ) is a drug commonly used to treat malaria. CQ has also gained interest for the treatment of other chronic diseases such as arthritis, lupus, cancer, diabetes, atherosclerosis, and dermatomyositis, among others. Since CQ is hydrophilic and low molecular-weight, attractive interactions with polymers in aqueous medium are weaker than with water, so that low encapsulation together with uncontrolled and fast release is observed. Importantly, a long-term administration of CQ is suggested, thus the development of formulations with controlled and prolonged release is desirable.

Results: Here we propose the use of aromatic interactions between the cationic CQ and the FDA approved polymer poly(sodium 4-styrenesulfonate) (PSS) for the formation and stabilization of nanoparticles (NPs). The strategy consists on the simple mixture of two aqueous solutions containing the oppositelly charged molecules. UV-vis and NMR spectroscopy evidence intimate aromatic-aromatic interactions between CQ and PSS. CQ/PSS molar ratios from 0.2 to 0.5 allow obtaining NPs with spherical shape, size in the range of $170-410 \mathrm{~nm}$, zeta potential from -18 to $-45 \mathrm{mV}$, and particles number in the range of 0.9 $6.6 \times 1010 \mathrm{NPs} / \mathrm{mL}$. Selected NPs (CQ/PSS molar ratio 0.4) are stable to wide variations in ionic strength (0-200 mM), $\mathrm{pH}(2-10)$ and temperature $\left(20-50^{\circ} \mathrm{C}\right)$. In addition, CQ/polymer 0.4 was also tested but with the absence of the aromatic group in the polymer, and providing smaller $(70 \mathrm{~nm})$, lower-concentrated (6.1 $x 109 \mathrm{NPs} / \mathrm{mL}$ ), and unstable particles, confirming the key role of the aromatic group. Furthermore, CQ/PSS NPs are stable during months and can be converted to a reconstitutable powder. Importantly, the selected NPs (CQ/PSS 0.4) show full drug association efficiency (100\%), very high drug loading (49\%), very high yield $(89 \%)$, and evidencing a drug entrapment/release governed by kinetic associations $(\approx 99$ $\%)$. Finally, release studies evidence a controlled and prolonged delivery.

Conclusions: Considering the potential uses of $\mathrm{CQ}$ for chronic diseases, and the simplicity and efficiency of our proposal, it could be considered as a valuable alternative to developed nanomedicines. In addition, this strategy could be used for other drugs and polymers showing similar characteristics to CQ and PSS.

\section{Introduction}

Chloroquine (CQ) is a cheap medicine that has been used worldwide for more than 70 years. It is part of the World Health Organization (WHO) model list of essential medicines and is primarily used to prevent and treat malaria [1-3]. Like most antimalarial agents, $C Q$ is currently widely used in autoimmune diseases such as severe rheumatoid arthritis [4-6] and systemic lupus erythematosus [7-9]. In addition, several investigations indicates its potential administration in the treatment of another chronic diseases (i.e. cancer [10, 11], diabetes [12, 13], atherosclerosis [14], dermatomyositis [15], and Sjögren's syndrome [16], among others). Several factors have influenced on the attention of $C Q$, highlighting their well-known immunomodulatory capacity [17].

Recently, several in vitro studies demonstrated that CQ efficiently inhibited the SARS-COV-2 infection, reason why this drug was purposed for the treatment of coronavirus disease 2019 (COVID-19). However, 
on June 15th the Food and Drug Administration (FDA) revoked the emergency use authorization for CQ (and their analogous hydroxychloroquine) to treat COVID-19 in United States of America [18]. This decision was supported on the evidence of several side effects that includes heart rhythm problems and others safety issues. Importantly, FDA mention that this decision does not affect the use of CQ for malaria and other autoimmune diseases. In this respect, $C Q$ is well tolerated by most people, even in longterm therapies [19]. This is supported due to their high capacity to bound plasmatic proteins and being eventually deposited into metabolically active tissues. Side effects as retinopathy, cardiomyopathy, myopathy, and neuromyopathy may could appear on prolonged treatments and high-level doses [20].

Considering the potential of this drug to treat several chronic diseases (malaria, arthritis, lupus, cancer, diabetes, atherosclerosis, dermatomyositis and SARS-COV-2, among others) and the appearance of side effects during prolonged treatments at high-level doses (retinopathy, cardiomyopathy, myopathy, and neuromyopathy) the development of formulations with controlled and prolonged drug release could represent a valuable alternative to optimize the efficacy, decrease side effects, and even to explore other uses.

Bearing in mind the physicochemical characteristics of CQ (cationic, hydrophilic, aromatic and low molecular-weight drug, Fig. 1, its entrapment in drug delivery systems such as nanoformulations to provide controlled and prolonged release, represents a main challenge [21-23]. The above statement is based on the very low capacity of this kind of molecules to undergo interactions with excipients that surpass their affinity with water, producing low drug association and, consequently, very fast drug release upon exposing the medicines to biological media [21-23]. In the state-of-the-art we could find some attempts to encapsulate CQ in nanocarriers. Among them, PLA [24], dextran [25], PEG [26], solid lipids [27], and PLGA [28] are described as encapsulant agents. In most cases, a drug burst release $(\approx 40 \%)$ was observed, and the complete release of CQ occurs within 1 to 3 days, thus not providing with controlled/prolonged drug release. We previously reported a green and highly efficient methodology to non-covalently attach three tricyclic drugs (imipramine, cyclobenzaprine, and amitriptyline), all of them hydrophilic and aromatic low molecular-weight molecules, to the hydrophilic and aromatic poly(sodium 4styrensulfonate) (PSS, Fig. 1) polymer. PSS is an approved excipient by the Food and Drug Administration (FDA) [29-33]. In that work we demonstrate that the formation of the NPs is based on ionic affinity and critically dependent on the occurrence of aromatic-aromatic interactions between the drug and the polymer.

The aim of this work is to provide a strategy based on aromatic-aromatic CQ-PSS interactions to create stable nanoformulations with high elaboration efficiencies (association efficiency, drug loading and yield) in addition to controlled and prolonged drug release. We also aim to evidence the critical role of the aromatic group in the selected polymer to form and stabilize de NPs at comparing formulations using a similar but non-aromatic polymer [poly (sodium vinylsulfonate)] (Fig. 1). Importantly, this study could be the basis to elaborate safer and more effective formulations to treat chronic diseases that require longterm therapies with CQ (or another hydrophilic, aromatic and low molecular-weight drug), such as malaria, rheumatoid arthritis, lupus erythematosus, diabetes mellitus, and SARS-COV-2, among others. 


\section{Results And Discussion}

\subsection{Elaboration and characterization of CQ/polymer formulations}

Based on previous investigations where our group demonstrated the synthesis of NPs comprising imipramine, amitriptyline, or cyclobenzaprine with the hydrophilic and aromatic polymer PSS [21], polymer already approved by the FDA for therapeutic applications [29, 30, 32, 34, 35], we postulate in this work a simple strategy to create NPs loaded with CQ.

First, we studied the binding between the only two components proposed to create the NPs (cationic CQ and the anionic polymers PSS or PVS (PVS is used as control due to the absence of the aromatic group). The UV-vis spectra of CQ (Fig. 2) exhibit strong absorptions attributed to $n-\pi^{*}$ transitions in the absence and presence of the selected polymers. In particular, a bathochromic effect is evident in the CQ spectrum in presence of PSS (Fig. 2C), showing a clear red shift that is typical for intimate aromatic - aromatic interactions (this effect is not observed for CQ in the presence of PVS) [36, 37]. In order to corroborate this observation, the ${ }^{1} \mathrm{H}-\mathrm{RMN}$ technique was selected considering the strong evidence demonstrated to identify changes in the chemical environment of protons by the presence of intimate aromatic-aromatic interactions [38-40]. In Fig. 3 can be observed the behavior of the aromatic protons of CQ in the absence (Fig. $3 \mathrm{~A}$ ) and the presence (Fig. $3 \mathrm{C}$ ) of PSS. As evidenced, the signals of the aromatic protons (between 6.0 and $8.0 \mathrm{ppm}$ ) of CQ are strongly affected by PSS and are upfield shifted together with a broadening of the signals (Fig. $3 \mathrm{C}$ ). This findings are characteristic for the presence of secondary site-specific aromatic-aromatic interactions since the hydration shell of the components changes, and the magnetic fields produced by electronic currents of aromatic groups strongly affect the neighbor structures [21, 41, 42]. A decrease on the molecular mobility is responsible for the broadening of the bands and the low signal-to-noise values.

Further, the formation of CQ/polymer NPs was evaluated. The strategy involves the simply mixing of two aqueous solutions (one containing the cationic drug $\mathrm{CQ}$ and the other containing the anionic aromatic or non-aromatic polymers, PSS and PVS, respectively) at room temperature, varying the drug concentration (between $3.3 \times 10^{-4} \mathrm{M}$ and $4.0 \times 10^{-3} \mathrm{M}$ ), and keeping constant the concentration of the polymers (PSS or PVS) at $3.3 \times 10^{-3} \mathrm{M}$. In this way, formulations with CQ/PSS molar ratio between 0.1 and 1.2 were developed. It is possible to observe in Fig. 4A that at lower ratio (CQ/PSS $\leq 0.3$ ) clear solutions are obtained which are attributed to the presence of soluble complexes and/or low concentrated NPs. At CQ/PSS $=0.4$ an highly opalescent and stable suspension is evidenced, presuming the formation of NPs showing high concentration; at higher ratio (CQ/PSS $\geq 0.5$ ), lower turbidity and the presence of macroprecipitates is evidenced, so that the colloidal state of the suspension collapses presumably due to a higher PSS charge neutralization by the CQ counterions. For CQ/PSS formulations, ionic (CQ and PSS are oppositely charged) and aromatic-aromatic interactions could reasonably contribute to the formation and stabilization of the systems. In the case of CQ/PVS formulations, significantly clearer suspensions 
are obtained (Fig. 4B) suggesting a lower magnitude of interactions between the two components. The absence of aromatic-aromatic drug-polymer interactions should influence this behavior.

In order to confirm our previous interpretation, the formulations were analyzed by dynamic light scattering (DLS) and laser doppler anemometry (LDA). As evidenced in Fig. 4C, CQ/PSS NPs were obtained from ratio 0.2 to $0.5(170-410 \mathrm{~nm})$, showing variable polydispersity ( $\mathrm{Pdl}$ in the range of $0.25-0.47)$, and evidencing that the main component in the surface of the NPs is the polymer PSS (-18 and $-45 \mathrm{mV})$. Other attempts for nanoencapsulation of CQ (using PLA, dextran, PEG, solid lipids or PLGA) reported hydrodynamic diameters around $50-500 \mathrm{~nm}$ with Pdl in the range of 0.07-0.35 [24-28]. Moreover, nanoparticle tracking analysis (NTA) evidenced nanoparticle concentrations in the range of $0.9 \times 10^{10}$ to $6.6 \times 10^{10} \mathrm{NPs} / \mathrm{mL}$ for our formulations. Other studies analyzing this parameter for amitriptyline-PSS NPs, doxorubicin loaded in carbomer NPs, and doxorubicin loaded in skim milk derived nanovesicles, reported values in the range of $1.0 \times 10^{6}-4.26 \times 10^{11} \mathrm{NPs} / \mathrm{mL}[21,43,44]$. Interestingly, when comparing formulations with PSS versus PVS, despite CQ/PVS also formed NPs at molar ratio 0.4 (presumably due to ionic bonds between the CQ and PVS), less efficient interactions are evidenced. This statement is reflected in the significantly smaller size $(\approx 70 \mathrm{~nm}$ for CQ/PVS versus $\approx 170 \mathrm{~nm}$ for CQ/PSS) together with the significantly lower concentrated particles (more than one order of magnitude less particles per $\mathrm{mL}$ ) when comparing with CQ/PSS 0.4, as evidenced in Figure S1 (supplementary information section). The above results confirm the importance of the presence of aromatic groups in the polymer to efficiently obtain NPs, and presumably also in their stabilization (see further studies in this paper). Considering the lower polydispersity (PDI 0.25) and higher nanoparticle concentration $\left(6.6 \times 10^{10} \mathrm{NPs} / \mathrm{mL}\right)$ obtained for CQ/PSS 0.4 , this formulation was selected to additional studies. The morphological structure of this formulation was characterized by scanning transmission electron microscope (STEM), as can be seen in Fig. 4D. The results showed uniform spherical structures with smooth surface. Interestingly, the size evidenced by STEM for this formulation (CQ/PSS 0.4) is smaller than that shown by DLS. These results could reflect the hydrated and partially swollen state of NPs during DLS studies; for STEM analysis, they shrink in the drying process during the sample preparation.

In order to investigate the stability of the obtained NPs (as a function of size and zeta potential), the formulations were subjected to different biological conditions and beyond, in addition to challenging storage conditions. Importantly, the results show that the CQ/PSS NPs are stable under all conditions (salt concentrations, Fig. 5A; pH gradient, Fig. 5B; temperature variations, Fig. 5C; and prolonged storage, Fig. 5D) with minimal deviations. Conversely, the instability presented by CQ/PVS 0.4 NPs in terms of salt concentrations, $\mathrm{pH}$ gradient and temperature variations (Fig. S2, see supplementary information) highlights the critical role of the aromatic group in the polymer to obtain stable NPs. Furthermore, freezedrying process was used to evaluate the transformation of the CQ/PSS nanoparticle suspension into a reconstitutable dry powder, thus facilitating the transport and storage while preventing biological contamination [45]. In Fig. 6, we can observe the achievement of optimal resuspension of the dry product, without significantly altering the size and zeta potential of the original formulations (fresh nanosuspension). Considering both the stability under physiological and storage conditions, and the 
possibility to convert the nanosuspension into a reconstitutable powder, the obtained CQ/PSS NPs are promising to postulate the formulation as a therapeutic alternative. Despite that in the literature we could find some investigations focused on identifying the stability of nanoformulations with CQ, our study demonstrates higher simplicity and stability (in suspension and as a powder), promoting this strategy, based on aromatic - aromatic interactions, as an attractive alternative to the state-of-the-art procedures.

\subsection{Association parameters, nature of the entrapment and release studies of CQ/PSS formulations}

In order to validate a selected nanoformulation for drug delivery purposes, the drug encapsulation parameters are critical. The association efficiency (AE), drug loading capacity and yield of CQ/PSS formulations were evaluated for all the range of tested ratios (CQ/PSS ratio between 0.1 and 1.2), and the results are shown in Fig. 7. Interestingly, it could be appreciated AE values in the range of $80-100 \%$ and drug loading values in the range of $15-49 \%$ for all tested formulations. Additionally, production yield values between $55-89 \%$ were obtained. Importantly, for the obtained CQ/PSS 0.4 formulation, an AE of $100 \%$, a drug loading of $49 \%$ and a yield of $89 \%$ was obtained. The very high values for these encapsulation parameters could be attributed to the high affinity between the CQ and the polymer PSS. These results are very promising since previous works for CQ loaded NPs comprising PLA, dextran, PEG, solid lipids or PLGA-tocopherol polyethylene glycol succinate reported AE values in the range of 64.1$99.9 \%$ [24-28], and drug loading in the range of $12.9-27.8 \%$ for PEG or solid lipid NPs [26, 27]. The success of our approach stands on the advantage of making intimate interactions (aromatic-aromatic interactions that presumably complement the ionic drug-polymer interactions) to bind the drug to the carrier. Thus, in this proposal, the drug is a structural component for the NPs formation, allowing the use of no more than one excipient to build the NPs, and requiring high load of the drug for this purpose.

Diafiltration studies were carried out to determine the nature of the drug entrapment/release, since the technique allows distinguishing kinetically bound molecules to molecules subjected to binding equilibrium (thermodynamically bound). The former are thought to place at the inner part of the NPs, stabilized in hydrophobic domains, and the latter are thought to bind into hydrated segments at the surface of the NPs. As previously reported, formulations with a higher kinetically bound fraction (\% KB) are associated with a controlled and prolonged release profile [21]. The diafiltration parameters for all tested CQ/PSS formulations (CQ/PSS between 0.1 and 1.2) are shown in Table S1 (see supplementary information). Importantly, the formulation CQ/PSS 0.4 presented very high values of kinetically bound molecules $(99.3 \pm 0.3 \%)$, thus reflecting a high content of CQ stabilized in the inner structure of the NPs. In addition, a low dissociation constant $\left(K^{\text {diss }} \approx 0.7\right)$ is found, reflecting the high tendency of this drug to bind the polymer, thus confirming the potential of the developed formulations and suggesting a controlled and prolonged release of CQ from the NPs.

To determine the release profile of the NPs CQ/PSS 0.4, conventional dialysis, and continuous dissolution studies in a USP 4 dissolution apparatus were selected as methodologies. Firstly, a sustained and prolonged release profile of CQ from the NPs in Milli-Q water was obtained. The cumulative drug release 
by dialysis as a function of time evidenced a maximum release of $3 \%$ in 30 days (Fig. 8A-a). In this condition, the binding between the aromatic drug CQ and the aromatic polymer PSS remains mainly inalterable and the release process is conditioned by the fraction of CQ molecules located on the NPS surface (thermodynamically bound) and consequently available to be released. Interestingly, under simulated biological conditions ( $\mathrm{pH} 7.4,0.13 \mathrm{M} \mathrm{NaCl}$ and $37^{\circ} \mathrm{C}$ ) a biphasic release profile was observed with an initial release phase (20\% in 6 hours), followed by a controlled and prolonged release phase until 30 days (Fig. 8A-a'). The observed controlled and prolonged release pattern represents a big improvement over other nanocarriers encapsulating CQ; dextran and PEG NPs showed a release profile that reaches 45-93\% during 3-8 $\mathrm{h}$ (analyzed by conventional dialysis) [25, 26], and PLA NPs show $40 \%$ of release during $10 \mathrm{~h}$ (analyzed by static Franz vertical diffusion cell) [24]. Furthermore, with the aim to study a more dynamic process, the continuous flow cell apparatus USP 4 was performed (Fig. 8B). As could be expected, a greater cumulative release of $16.2 \%$ in the first 6 hours was obtained, evidencing that the exposition of these NPs to a continuous flux of medium accelerates the release of CQ.

Finally, the release mechanism obtained by the fit of the data to different mathematical models (i.e., zero order, first order, Higuchi and Korsmeyer-Peppas) was evaluated. The Korsmeyer-Peppas fitted better the experimental data (Table S2, see supplementary information). Considering the characteristics of the components (hydrophilic molecules), and that CQ is a structural component of the NPs, the drug release process should involve the release of CQ molecules from the surface of the NPs, due to equilibrium displacement, and followed by hydration and rearrangement of the boundary between the inner and the surface of the NPs. The detachment of the drug from the NP enhances the hydration of the system, so that the subjacent confined drug molecules achieve a hydrated environment and establishing equilibrium with the bulk. This process proceeds in a cyclic manner. This complex process is consistent with deviations of the linear or first order behavior of the release as a function of time.

\section{Conclusions}

In conclusion, our results offer an efficient strategy to nanoencapsulate chloroquine (CQ) in nanocarriers. The strategy involves taking advantage of aromatic-aromatic interactions between CQ and PSS (as a unique excipient) for the formation and stabilization of the NPs. CQ/PSS NPs are stable to variations in ionic strength, $\mathrm{pH}$, and temperature. Conversely, a similar formulation but with the absence of the aromatic ring in the polymer (testing ionic-ionic drug-polymer interactions for NPs formation and stabilization), provided smaller, low-concentrate, and unstable particles, confirming the key role of the aromatic group in the polymer. In addition, CQ/PSS NPs are stable at storage conditions and can be easily freeze-dried and reconstituted in water. Furthermore, the selected formulation of NPs shows a full drug association efficiency $(\approx 100 \%)$, very high drug loading $(\approx 49 \%)$, and very high yield $(\approx 89 \%)$. In addition, it was shown that the drug entrapment is governed mainly by kinetic interactions $(\approx 99 \%)$, and being the drug mostly confined at the inner of the NPs. Finally, using different release methodologies, we have demonstrated that CQ/PSS NPs offer a controlled and prolonged drug release profile. Considering the wide therapeutic possibilities for $C Q$ in the treatment of a variety of chronic diseases, and the advantages that our technology offers in comparison with other approaches (being simple, cheap, 
efficient, and low-pollutant), the proposed strategy could represent the basis for a medicine with controlled/prolonged release to treat different pathologies where this release pattern of CQ is required (malaria, rheumatoid arthritis, lupus erythematosus, and diabetes, among others). In addition, this strategy could be used for other drugs and polymers showing similar characteristics to CQ and PSS.

\section{Materials And Methods}

\subsection{Materials}

Chloroquine diphosphate $(515.9 \mathrm{~g} / \mathrm{mol}$ ) was purchased from Sigma Aldrich (USA) and was used as received. The anionic aromatic polymer poly(sodium 4-styrenesulfonate) (PSS) $(206.2 \mathrm{~g} / \mathrm{mol}$ of monomeric units) was purchased from Sigma Aldrich (USA) and purified and fractionated by diafiltration over a membrane of a molecular weight cut-off (MWCO) of $5000 \mathrm{Da}$ (Biomax, $63.5 \mathrm{~mm}$ diameter), first in the presence of $0.15 \mathrm{M} \mathrm{NaNO}_{3}$ (Sigma Aldrich, USA) and then in the absence of the electrolyte. The anionic aliphatic polymer poly (sodium vinylsulfonate) (PVS) $(130.1 \mathrm{~g} / \mathrm{mol}$ of monomeric units) was purchased from Sigma Aldrich (USA) and was used as received. The $\mathrm{pH}$ was adjusted with an $\mathrm{Edge}{ }^{\circledR}$ $\mathrm{HI} 2002 \mathrm{pH}$ meter (Hanna Instruments, USA) with minimum amounts of $\mathrm{NaOH}$ and $\mathrm{HCl}$ (Merck, Germany). All the solutions were prepared with Milli-Q water obtained from a Simplicity SIMS 00001 equipment (Millipore, France). The structure of CQ and each polymer (PSS and PVS) are shown in Fig. 1.

\subsection{Methods}

\subsubsection{UV-vis and NMR spectroscopy between CQ and polymers}

UV-vis experiments were performed in an Agilent 8453 spectrophotometer (Agilent, USA) using a quartz cuvette (Hellma ${ }^{\circledR}$, Germany) of $1 \mathrm{~cm}$ of path length. For the experiments, $1 \mathrm{~mL}$ of [CQ] $=1.3 \times 10^{-5} \mathrm{M}$ in the absence of polymers, and in the presence of $[\mathrm{PSS}]=3.3 \times 10^{-5} \mathrm{M}$ or $[\mathrm{PVS}]=3.3 \times 10^{-5} \mathrm{M}$ was used. NMR analyses were performed in $\mathrm{D}_{2} \mathrm{O}$ on an Avance 400 (Bruker, USA) using glass tubes of $5 \mathrm{~mm}$ diameter (volume of solution typically $0.7 \mathrm{~mL}$ ). Appropriate conditions for ${ }^{1} \mathrm{H}$-NMR experiments were chosen as [CQ] $=1 \times 10^{-3} \mathrm{M}$ and [PSS] $=1 \times 10^{-2} \mathrm{M}$, a volume of $750 \mu \mathrm{L}$ of each compound was lyophilized individually and reconstituted in $\mathrm{D}_{2} \mathrm{O}$ before analysis.

\subsubsection{Preparation of $\mathrm{CQ} /$ polymer formulations}

The CQ/polymer formulations were synthetized according to the method previously reported by our group [21]. Briefly, $5.0 \mathrm{~mL}$ of an aqueous solution containing $\mathrm{CQ}$ was added to $5.0 \mathrm{~mL}$ of an aqueous solution containing the anionic polymers (PSS and PVS), at pH 7, and exposed to continuous stirring ( $5 \mathrm{~min}$ ). The final apparent concentration was defined in order to obtain different molar ratios defined as [CQ]/[polymer]. The CQ concentration was varied between $3.3 \times 10^{-4} \mathrm{M}$ and $4.0 \times 10^{-3} \mathrm{M}$ and the anionic 
polymer concentration was constant at $3.3 \times 10^{-3} \mathrm{M}$. The formation (or not) of NPs was evidenced through dynamic light scattering (DLS).

\subsubsection{Physicochemical characterization of the nanoparticles}

The hydrodynamic diameter and zeta potential of the formulations were determined by dynamic light scattering (DLS) and laser Doppler anemometry (LDA) using a ZetaSizer NanoZS (Malvern Instruments, UK).

The determination of nanoparticle concentration was performed in a NanoSight NS300 (Malvern Instruments, UK). The samples were diluted up to 100 times with Milli-Q water to achieve an optimum concentration range of $10^{7}-10^{9}$ particles $/ \mathrm{mL}$. A minimum of five videos (one minute each one) of the particles moving under Brownian motion were captured by the NanoSight. The videos were then analyzed for size distribution and particle concentration using the built-in NTA v 3.0 software (Malvern, UK).

The morphological characterization was carried out in a scanning transmission electron microscope (STEM), model Inspect F-50 (FEl, Holland). STEM images were obtained by sticking a droplet (20 $\mu \mathrm{L})$ of the formulation to a copper grid (200 mesh, covered with Formvar) for 2 min, then removing the droplet with filter paper avoiding the paper touching the grid, then washing the grid twice with a droplet of Milli-Q water for $1 \mathrm{~min}$ and removing the droplet with filter paper. Subsequently, the sample was stained with a solution of $1 \%(\mathrm{w} / \mathrm{v})$ phosphotungstic acid by adding a droplet of this solution to the grid for 2 min and then removing with filter paper. Finally, the grid was dried at room temperature for at least $1 \mathrm{~h}$ before being analyzed.

\subsubsection{Drug association efficiency, drug loading, and yield of the CQ/PSS formulations}

Drug association efficiency, drug loading, and yield were obtained as previously described [21, 45]. Briefly, the association efficiency of CQ in the CQ/PSS formulations was determined by analyzing the ratio between the amount of drug associated in the formulation and the total initial drug (associated and nonassociated). The drug loading (\% w/w) was calculated by dividing the amount of drug associated by the total weight of the formulations. The yield was calculated by dividing the total final weight of each formulation by the total initial weight of the components (CQ + PSS). The drug content into the formulations was calculated indirectly by quantifying the free drug in the medium; the separation of NPs and free drug was done by using Vivaspinß6 tubes (MWCO 3 kDa, 5000 G x 40 min). The quantification of the CQ was done by measuring the absorbance at $343 \mathrm{~nm}$ (Agilent 8453 spectrophotometer, USA), respectively. The standard curve of $C Q$ was linear $\left(R^{2}>0.999\right)$ in the range of concentrations between $4 \times$ $10^{-5} \mathrm{M}$ and $3 \times 10^{-6} \mathrm{M}$ (molar extinction coefficient was $30449 \mathrm{M}^{-1} \mathrm{~cm}^{-1}$ ). Finally, for the calculation of the total final weight, $1 \mathrm{~mL}$ of each formulation was lyophilized in glass vials, which were weighed before adding the formulation and after freeze-drying to assess the total solid mass (glass vials + formulation). The lyophilization procedure was done in the freeze-dryer equipment FreeZone 1 (Labconco, USA) using a high vacuum pump (50 mTorr) for $24 \mathrm{~h}$. 


\subsubsection{Kinetic and/or thermodynamic drug entrapment, and dissociation constants evaluated by diafiltration}

The diafiltration method was selected to investigate the kinetic and/or thermodynamic drug entrapment, and the dissociation constants of their equilibrium binding to excipients/formulations [36, 37, 46-53]. The unit used for diafiltration analyses consisted on a diafiltration cell (10 mL, Amicon 8010), a regenerated cellulose membrane (cutoff of 5000 Dalton, Merck, Germany), a reservoir, a selector, and a pressure source (Merck-Millipore, Germany). The method consists on passing through the diafiltration cell containing the formulation of NPs in water, a continuous liquid supply from the donor chamber (reservoir) keeping a constant volume in the diafiltration cell. A two-compartment system model is considered for data treatment [49]. For the diafiltration experiments, aliquots of $10 \mathrm{~mL}$ of the formulations were added into the diafiltration cell and then filtered under 3 bars of pressure and magnetic stirring. Milli-Q water $(\mathrm{pH}$ 7) was used as solvent for diafiltration. A total of 8 samples (approx. $5 \mathrm{~mL}$ ) were collected and the concentration of $C Q$ in each sample was determined by spectrophotometry $[21,53]$.

In this paper, diafiltration was performed to determine the fraction of CQ kinetically or thermodynamically bound to PSS. Details of diafiltration procedures, mathematical analysis and results are widely explained in previous studies [36, 37, 46-53]. Briefly, the parameters $v$ and $u$ represent the initial fraction of CQ thermodynamically bound to the particles, thus in equilibrium, and the initial fraction of drug bound to the nanoparticles whose release is kinetically controlled, respectively. The parameter $j$ is related to the strength of interaction corresponding to the reversibly bound drug fraction $(v)$. The parameters $u^{m}$ and $k^{m}$ correspond to $u$ and $j$ values, respectively, obtained in blank experiments as controls, performed by diafiltration of the drug in the absence of other excipients or formulations.

Subsequently, the thermodynamically bound $(T B)$ and kinetically bound $(K B)$ fractions were determined as follows:

$T B=v\left(k^{m}-j\right) / k^{m}(1)$

$K B=u-u^{m}(2)$

In addition, the dissociation constants ( $\left.K^{\text {diss }} \equiv c^{\text {free }} / c^{\text {rev-bound }}\right)$, where $c^{\text {rev-bound }}$ is the concentration of drug reversibly bound to the matrix, and $c^{\text {free }}$ is the concentration of drug free in the bulk, could be also determined by diafiltration following the Eq. (3).

$j /(1-j) \leq K^{d i s s} \leq k^{m} j / k^{m}-j(3)$

\subsubsection{In-vitro drug release studies}

In-vitro drug release assays were carried out using two different methods: conventional dialysis and USP apparatus 4 (continuous flow-cell). 


\subsubsection{Dialysis:}

$5 \mathrm{~mL}$ of CQ/PSS formulations were added in a dialysis bag (MWCO $10 \mathrm{kDa}$, ThermoScientific, USA). The dialysis system was immersed in $95 \mathrm{~mL}$ of Milli-Q water $(\mathrm{pH}$ 7.0) or simulating biological conditions (Milli-Q water, $0.13 \mathrm{M} \mathrm{NaCl}, \mathrm{pH} 7.4$ ), and kept at $37^{\circ} \mathrm{C}$ and continuous agitation (C-MAG HS 7, IKA, Staufen, Germany). The experiments were carried out until 30 days, aliquots (500 $\mu \mathrm{L})$ of the solution were withdrawn at certain time intervals and replaced with an equal volume of fresh release medium. The amount of released CQ was determined by measuring the absorbance of each aliquot by spectrophotometry (Agilent 8453 spectrophotometer, USA).

\subsubsection{USP apparatus 4:}

For this assay, the set-up of the continuous flow method is combined with a dialysis membrane to contain the nanoformulations into the cell [54]. In brief, $5 \mathrm{~mL}$ of CQ/PSS NPs were added in a dialysis bag (MWCO $10 \mathrm{kDa}$, ThermoScientific, USA) and then immersed into the flow-cell (12.5 $\mathrm{mL}$ capacity). Drug release studies were assessed using $250 \mathrm{~mL}$ of Milli-Q water at $\mathrm{pH}$ 7. The continuous flow-cell (Sotax CE 6, Sotax AG, Switzerland) is operated in close configuration at $37^{\circ} \mathrm{C}$ and with a flow rate of 4 $\mathrm{mL} / \mathrm{min}$ approximately. The experiment was carried out for 6 hours, aliquots $(500 \mu \mathrm{L})$ of the solution were withdrawn every $15 \mathrm{~min}$ and replaced with an equal volume of fresh Milli-Q water. The amount of released CQ was determined by measuring the absorbance of each aliquot by spectrophotometry (Agilent 8453 spectrophotometer, USA).

To investigate the release mechanism (in both conventional dialysis and the USP apparatus 4 data), mathematical kinetics modelling was done using the program DDSolver [55]. The coefficient of determination $\left(R^{2}\right)$, the Akaike information criteria (AIC), and the model selection criteria (MSC) parameters were considered for the model selection. Finally, the release data was fitted to zero order, first order, Higuchi and Korsmeyer-Peppas [56].

\section{Declarations}

\subsection{Ethics approval and consent to participate}

Not applicable

\subsection{Consent for publication}

Not applicable

\subsection{Availability of data and materials}


All data generated or analysed during this study are included in this published article [and its supplementary information files].

\subsection{Competing interests}

The authors declare that they have no competing interests

\subsection{Funding}

This work was supported by FONDECYT 1201899 (F.A.O-A.), FONDECYT 1181695 (I.M-V.), ANID/PIA/ACT192144 (F A. O-A) and FONDAP 15130011.

\subsection{Authors' contributions}

M.G.V-S. developed the experimental section and contributed with the writing of the manuscript. I.M-V and F.A.O-A guided all the experimental section and contribute with the writing of the manuscript.

\subsection{Acknowledgements}

Not applicable

\section{References}

1. Conrad, M.D. and P.J. Rosenthal, Antimalarial drug resistance in Africa: the calm before the storm? The Lancet Infectious Diseases, 2019. 19(10): p. e338-e351.

2. Schlagenhauf, P. and K.C. Kain, 15 - Malaria Chemoprophylaxis, in Travel Medicine (Third Edition), J.S. Keystone, et al., Editors. 2013, Content Repository Only!: London. p. 143-162.

3. Schlagenhauf, P., et al., 15 - Malaria Chemoprophylaxis, in Travel Medicine (Fourth Edition), J.S. Keystone, et al., Editors. 2019, Elsevier: London. p. 145-167.

4. dos Reis Neto, E.T., et al., Revisiting hydroxychloroquine and chloroquine for patients with chronic immunity-mediated inflammatory rheumatic diseases. Advances in Rheumatology, 2020. 60(1): p. 32.

5. Bjelle, A., et al., Chloroquine in long-term treatment of rheumatoid arthritis. Clin Rheumatol, 1983. 2(4): p. 393-9.

6. Schrezenmeier, E. and T. Dörner, Mechanisms of action of hydroxychloroquine and chloroquine: implications for rheumatology. Nature Reviews Rheumatology, 2020. 16(3): p. 155-166.

7. Fanouriakis, A., G.K. Bertsias, and D.T. Boumpas, Chloroquine as alternative antimalarial in systemic lupus erythematosus. Response to '2019 update of the EULAR recommendations for the 
management of SLE: don't forget chloroquine' by Figueroa-Parra <em>et al</em>. 2020. 79(9): p. e115-e115.

8. Lupus_Foundation_of_America. Why are treatments that were developed for malaria now used for lupus? 2020; Available from: https://www.lupus.org/resources/why-are-treatments-that-weredeveloped-for-malaria-now-used-for-lupus.

9. Lee, S.J., E. Silverman, and J. Bargman, The role of antimalarial agents in the treatment of SLE and lupus nephritis. Nature reviews. Nephrology, 2011. 7: p. 718-29.

10. Zhang, Y., et al., Chloroquine (CQ) exerts anti-breast cancer through modulating microenvironment and inducing apoptosis. International Immunopharmacology, 2017. 42: p. 100-107.

11. Verbaanderd, C., et al., Repurposing Drugs in Oncology (ReDO)-chloroquine and hydroxychloroquine as anti-cancer agents. Ecancermedicalscience, 2017. 11: p. 781-781.

12. Hage, M.P., M.R. Al-Badri, and S.T. Azar, A favorable effect of hydroxychloroquine on glucose and lipid metabolism beyond its anti-inflammatory role. Therapeutic advances in endocrinology and metabolism, 2014. 5(4): p. 77-85.

13. de Almeida Júnior, R.F., et al., Chloroquine as a promising adjuvant therapy for type 1 Diabetes Mellitus. Scientific Reports, 2020. 10(1): p. 12098.

14. Shukla, A.M., et al., Impact of Hydroxychloroquine on Atherosclerosis and Vascular Stiffness in the Presence of Chronic Kidney Disease. PloS one, 2015. 10(9): p. e0139226-e0139226.

15. Anyanwu, C.O., et al., The systemic management of cutaneous dermatomyositis: Results of a stepwise strategy. International Journal of Women's Dermatology, 2017. 3(4): p. 189-194.

16. Lee, H.J., et al., The Effect of Chloroquine on the Development of Dry Eye in Sjögren Syndrome Animal Model. Investigative Ophthalmology \& Visual Science, 2019. 60(12): p. 3708-3716.

17. Ben-Zvi, I., et al., Hydroxychloroquine: from malaria to autoimmunity. Clin Rev Allergy Immunol, 2012. 42(2): p. 145-53.

18. Food_and_Drug_Administration_(FDA). FDA cautions against use of hydroxychloroquine or chloroquine for COVID-19 outside of the hospital setting or a clinical trial due to risk of heart rhythm problems. 2020 July 1, 2020; Available from: https://www.fda.gov/drugs/drug-safety-andavailability/fda-cautions-against-use-hydroxychloroquine-or-chloroquine-covid-19-outside-hospitalsetting-or.

19. Wilson, C.M., 296 - Antiparasitic Agents, in Principles and Practice of Pediatric Infectious Diseases (Fourth Edition), S.S. Long, Editor. 2012, Content Repository Only!: London. p. 1518-1545.e3.

20. Braga, C.B.E., et al., Side Effects of Chloroquine and Primaquine and Symptom Reduction in Malaria Endemic Area (Mâncio Lima, Acre, Brazil). Interdisciplinary perspectives on infectious diseases, 2015. 2015: p. 346853-346853.

21. Villamizar-Sarmiento, M.G., et al., A New Methodology to Create Polymeric Nanocarriers Containing Hydrophilic Low Molecular-Weight Drugs: A Green Strategy Providing a Very High Drug Loading. Molecular Pharmaceutics, 2019. 16(7): p. 2892-2901. 
22. Varghese, O.P., et al., Chondroitin sulfate derived theranostic nanoparticles for targeted drug delivery. Biomaterials Science, 2016. 4(9): p. 1310-1313.

23. Jin, X., et al., Supramolecular nanoscale drug-delivery system with ordered structure. National Science Review, 2019. 6(6): p. 1128-1137.

24. Lima, T.L.C., et al., Improving Encapsulation of Hydrophilic Chloroquine Diphosphate into Biodegradable Nanoparticles: A Promising Approach against Herpes Virus Simplex-1 Infection. Pharmaceutics, 2018. 10(4): p. 255.

25. Kashyap, A., et al., Chloroquine diphosphate bearing dextran nanoparticles augmented drug delivery and overwhelmed drug resistance in Plasmodium falciparum parasites. Int J Biol Macromol, 2018. 114: p. 161-168.

26. Usman, M. and M. Akhyar Farrukh, Delayed release profile of iron nano-chloroquine phosphate and evaluation of its toxicity. Materials Today: Proceedings, 2018. 5(7, Part 3): p. 15645-15652.

27. Muga, J.O., et al., In vitro evaluation of chloroquine-loaded and heparin surface-functionalized solid lipid nanoparticles. Malaria Journal, 2018. 17(1): p. 133.

28. Sun, J.H., et al., Co-delivery nanoparticles of doxorubicin and chloroquine for improving the anticancer effect in vitro. Nanotechnology, 2019. 30(8): p. 085101.

29. Palaka, E., et al., Evidence in support of hyperkalaemia management strategies: A systematic literature review. International Journal of Clinical Practice, 2018. 72(2).

30. Lepage, L., et al., Randomized Clinical Trial of Sodium Polystyrene Sulfonate for the Treatment of Mild Hyperkalemia in CKD. Clinical Journal of the American Society of Nephrology, 2015. 10(12): p. 2136-2142.

31. Lim, H.-P., et al., Controlled delivery of oral insulin aspart using $\mathrm{pH}$-responsive alginate/ $\mathrm{K}$-carrageenan composite hydrogel beads. Reactive and Functional Polymers, 2017. 120: p. 20-29.

32. Mistry, M., et al., Evaluation of Sodium Polystyrene Sulfonate Dosing Strategies in the Inpatient Management of Hyperkalemia. Annals of Pharmacotherapy, 2016. 50(6): p. 455-462.

33. Wu, C., et al., Redox-responsive supramolecular hydrogel based on 10-hydroxy camptothecin-peptide covalent conjugates with high loading capacity for drug delivery. Materials Science and Engineering: C, 2017. 76: p. 196-202.

34. Hunt, T.V., et al., Single-dose sodium polystyrene sulfonate for hyperkalemia in chronic kidney disease or end-stage renal disease. Clinical Kidney Journal, 2018.

35. Hollander-Rodriguez, J.C. and J.F. Calvert, Jr., Hyperkalemia. Am Fam Physician, 2006. 73(2): p. 28390.

36. Moreno-Villoslada, l., et al., $\pi$-Stacking of rhodamine $B$ onto water-soluble polymers containing aromatic groups. Polymer, 2006. 47(19): p. 6496-6500.

37. Moreno-Villoslada, I., et al., Comparative study of the self-aggregation of rhodamine $6 G$ in the presence of poly(sodium 4-styrenesulfonate), poly(N-phenylmaleimide-co-acrylic acid), poly(styrenealt-maleic acid), and poly(sodium acrylate). J Phys Chem B, 2010. 114(37): p. 11983-92. 
38. Sun, H., et al., The pi-pi stacked geometries and association thermodynamics of quinacridone derivatives studied by 1 H NMR. J Phys Chem A, 2006. 110(37): p. 10750-6.

39. Parenti, F., et al., П-Stacking Signature in NMR Solution Spectra of Thiophene-Based Conjugated Polymers. ACS Omega, 2017. 2(9): p. 5775-5784.

40. Masoodi, H.R. and S. Bagheri, Interplay between $\pi \cdots \pi$ stacking and cation $\cdots \pi$ interaction: a theoretical NMR study. Journal of the Iranian Chemical Society, 2015. 12(10): p. 1883-1892.

41. Zhao, L., H. Zhang, and W. Wang, $\pi-\pi$ stacking interaction in mixed surfactant solutions assembled by cationic surfactant and organic salt with a naphthalene nucleus. Journal of Molecular Liquids, 2017. 240: p. 14-20.

42. Nageh, H., Y. Wang, and T. Nakano, Cationic polymerization of dibenzofulvene leading to a $\pi$-stacked polymer. Polymer, 2018. 144: p. 51-56.

43. Sintov, A.C. and G. Enden, New doxorubicin nanoparticles engineered from calcium-crosslinked carbomer and a microemulsion precursor. Drug Development and Industrial Pharmacy, 2017. 43(5): p. 830-838.

44. George, J., I.K. Yan, and T. Patel, Nanovesicle-mediated delivery of anticancer agents effectively induced cell death and regressed intrahepatic tumors in athymic mice. Lab Invest, 2018. 98(7): p. 895-910.

45. Oyarzun-Ampuero, F.A., et al., Hyaluronan nanocapsules as a new vehicle for intracellular drug delivery. Eur J Pharm Sci, 2013. 49(4): p. 483-90.

46. Fuenzalida, J.P., et al., Immobilization of hydrophilic low molecular-weight molecules in nanoparticles of chitosan/poly(sodium 4-styrenesulfonate) assisted by aromatic-aromatic interactions. J Phys Chem B, 2014. 118(32): p. 9782-91.

47. Moreno-Villoslada, I., et al., Aromatic-aromatic interaction between 2,3,5-triphenyl-2H-tetrazolium chloride and poly(sodium 4-styrenesulfonate). J Phys Chem B, 2007. 111(22): p. 6146-50.

48. Moreno-Villoslada, I., et al., Interactions of 2,3,5-triphenyl-2H-tetrazolium chloride with poly(sodium 4styrenesulfonate) studied by diafiltration and UV-vis spectroscopy. Journal of Membrane Science, 2004. 244(1-2): p. 205-213.

49. Moreno-Villoslada, I., et al., Simultaneous interactions between a low molecular-weight species and two high molecular-weight species studied by diafiltration. Journal of Membrane Science, 2006. 272(1-2): p. 137-142.

50. Moreno-Villoslada, I., et al., Comparison between the binding of chlorpheniramine maleate to poly(sodium 4-styrenesulfonate) and the binding to other polyelectrolytes. Polymer, 2005. 46(18): p. 7240-7245.

51. Moreno-Villoslada, I., et al., Binding of chlorpheniramine maleate to pharmacologically important alginic acid, carboxymethylcellulose, $k$-carageenan, and l-carrageenan as studied by diafiltration. Journal of Applied Polymer Science, 2005. 98(2): p. 598-602.

52. Moreno-Villoslada, I., et al., Stacking of 2,3,5-triphenyl-2H-tetrazolium chloride onto polyelectrolytes containing 4-styrenesulfonate groups. J Phys Chem B, 2008. 112(36): p. 11244-9. 
53. Orellana, S.L., et al., Association Efficiency of Three Ionic Forms of Oxytetracycline to Cationic and Anionic Oil-In-Water Nanoemulsions Analyzed by Diafiltration. Journal of Pharmaceutical Sciences, 2015. 104(3): p. 1141-1152.

54. Nothnagel, L. and M.G. Wacker, How to measure release from nanosized carriers? European Journal of Pharmaceutical Sciences, 2018. 120: p. 199-211.

55. Zhang, Y., et al., DDSolver: an add-in program for modeling and comparison of drug dissolution profiles. The AAPS journal, 2010. 12(3): p. 263-271.

56. Catalan-Figueroa, J., et al., A mechanistic approach for the optimization of loperamide loaded nanocarriers characterization: Diafiltration and mathematical modeling advantages. European Journal of Pharmaceutical Sciences, 2018. 125: p. 215-222.

\section{Figures}

Figure 1

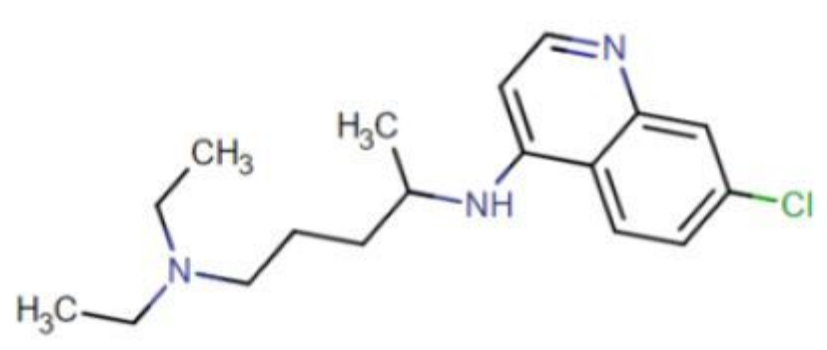

Chloroquine<smiles>CC(C)(C)C(c1ccc(S(=O)(=O)O)cc1)C(C)(C)C</smiles>

Poly(4-styrenesulfonate)

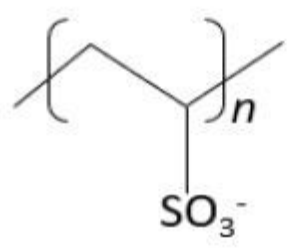

Poly(vinylsulfonate)

\section{Figure 1}

Molecular structures 
Figure 2

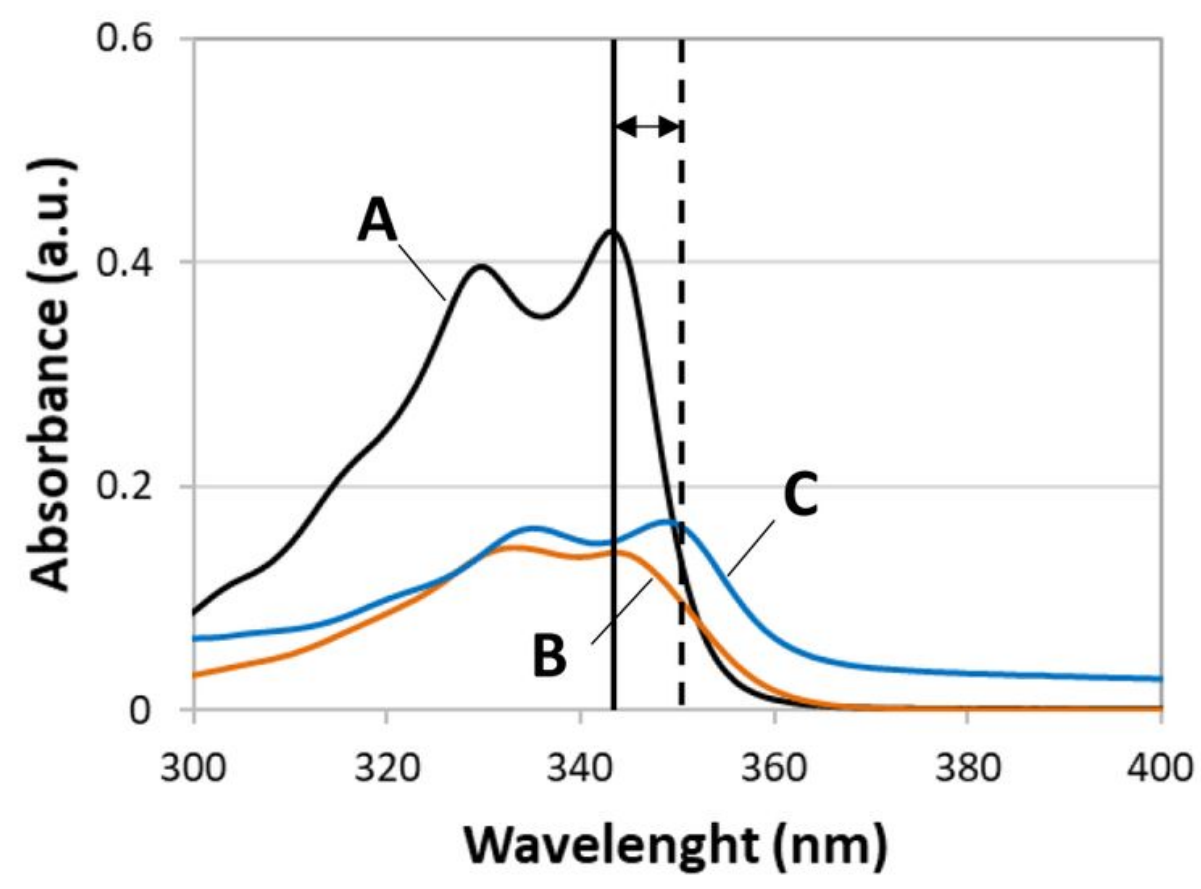

Figure 2

UV-vis spectra of CQ $1.3 \times 10-5 \mathrm{M}$ in the absence of polymers (A), CQ $1.3 \times 10-5 \mathrm{M}$ in presence of PVS 3.3 $x$ 10-5 M (B) and CQ $1.3 \times 10-5$ in presence of PSS $3.3 \times 10-5 \mathrm{M}(\mathrm{C})$. 
Figure 3
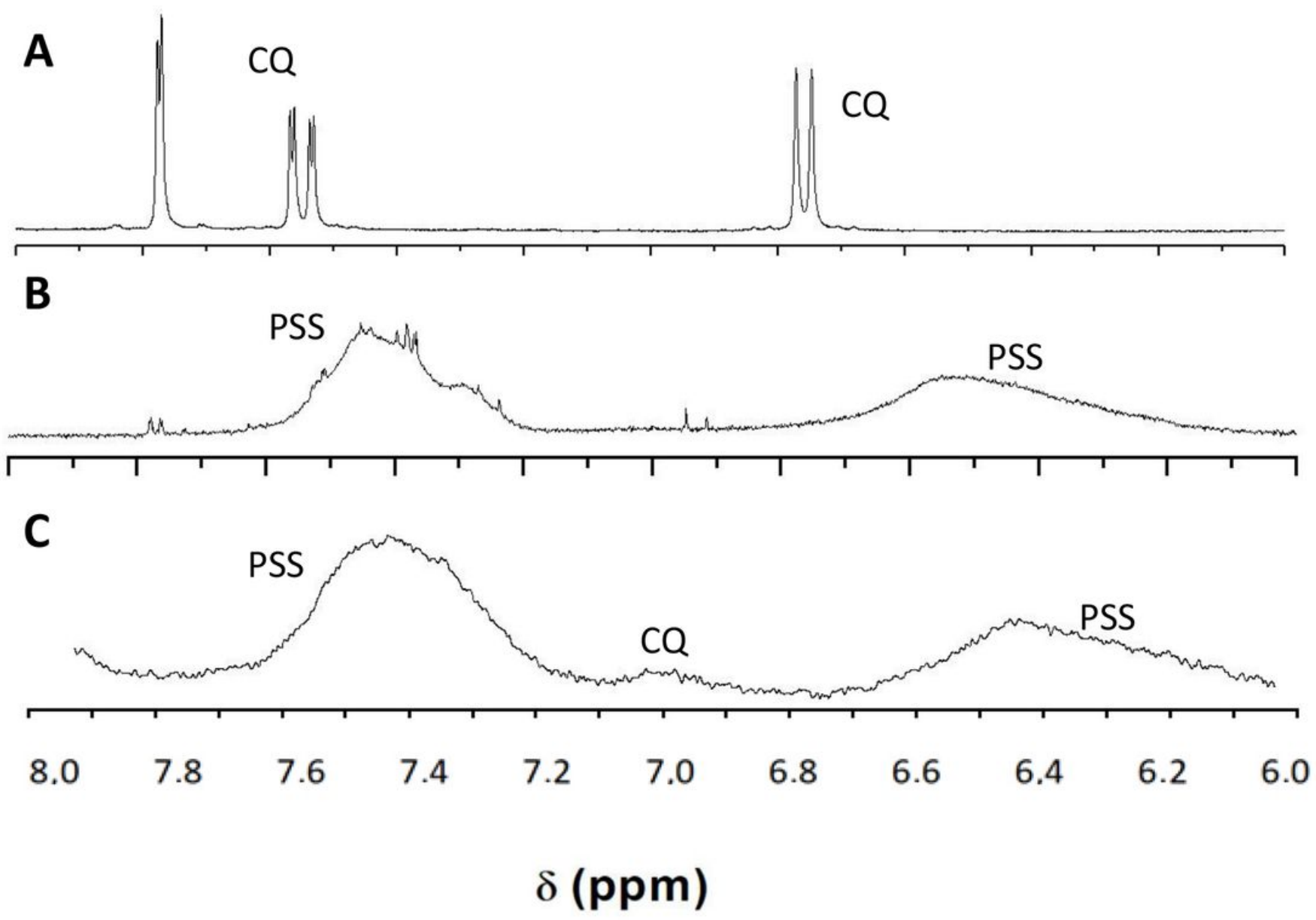

Figure 3

$1 \mathrm{H}-\mathrm{NMR}$ spectra $(500 \mathrm{MHz})$ in $\mathrm{D} 20$ at $\mathrm{pH} 7$ of the aromatic region of solutions containing: CQ $1 \times 10-3 \mathrm{M}$ in the absence of PSS (A), PSS $1 \times 10-2 \mathrm{M}(\mathrm{B})$, and CQ $1 \times 10-3 \mathrm{M}$ in the presence of PSS $1 \times 10-2 \mathrm{M}(\mathrm{C})$. 
Figure 4
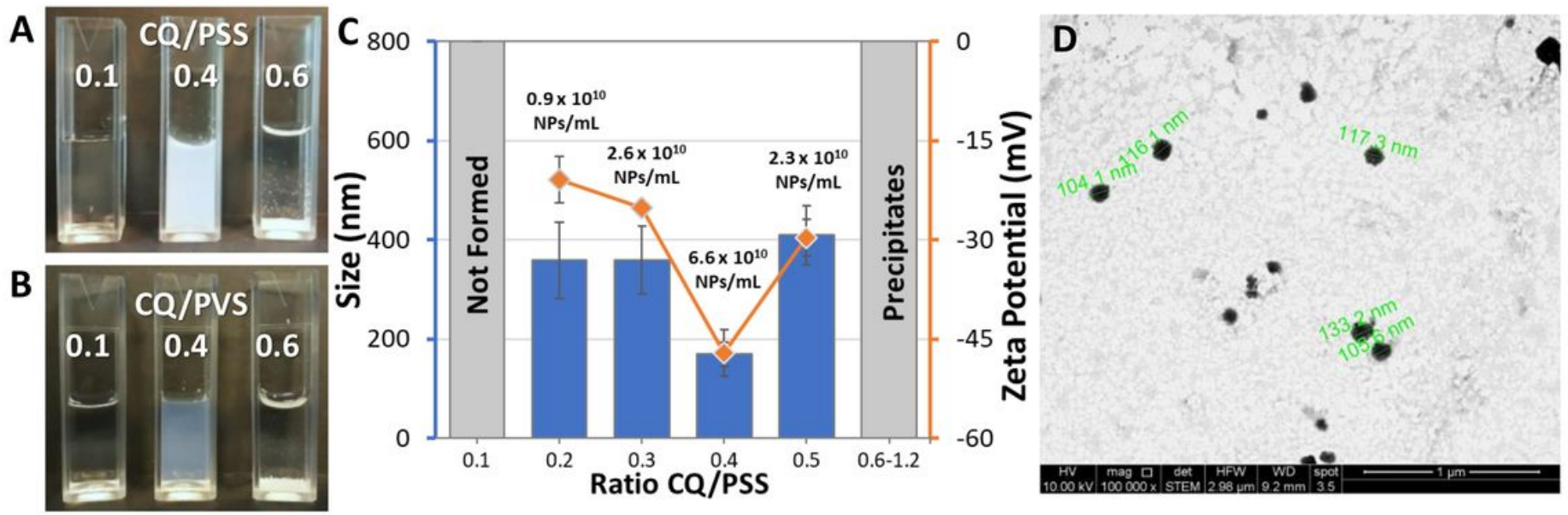

Figure 4

Optical images of CQ/PSS 0.1, 0.4, 0.6 (A) and CQ/PVS 0.1, 0.4, 0.6 (B). Size (bars), zeta potential (rhombuses) and NPs concentration (number) of tested CQ/PSS formulations (mean \pm SD; $n=3)(C)$. STEM image of NPs CQ/PSS 0.4 (D).

Figure 5
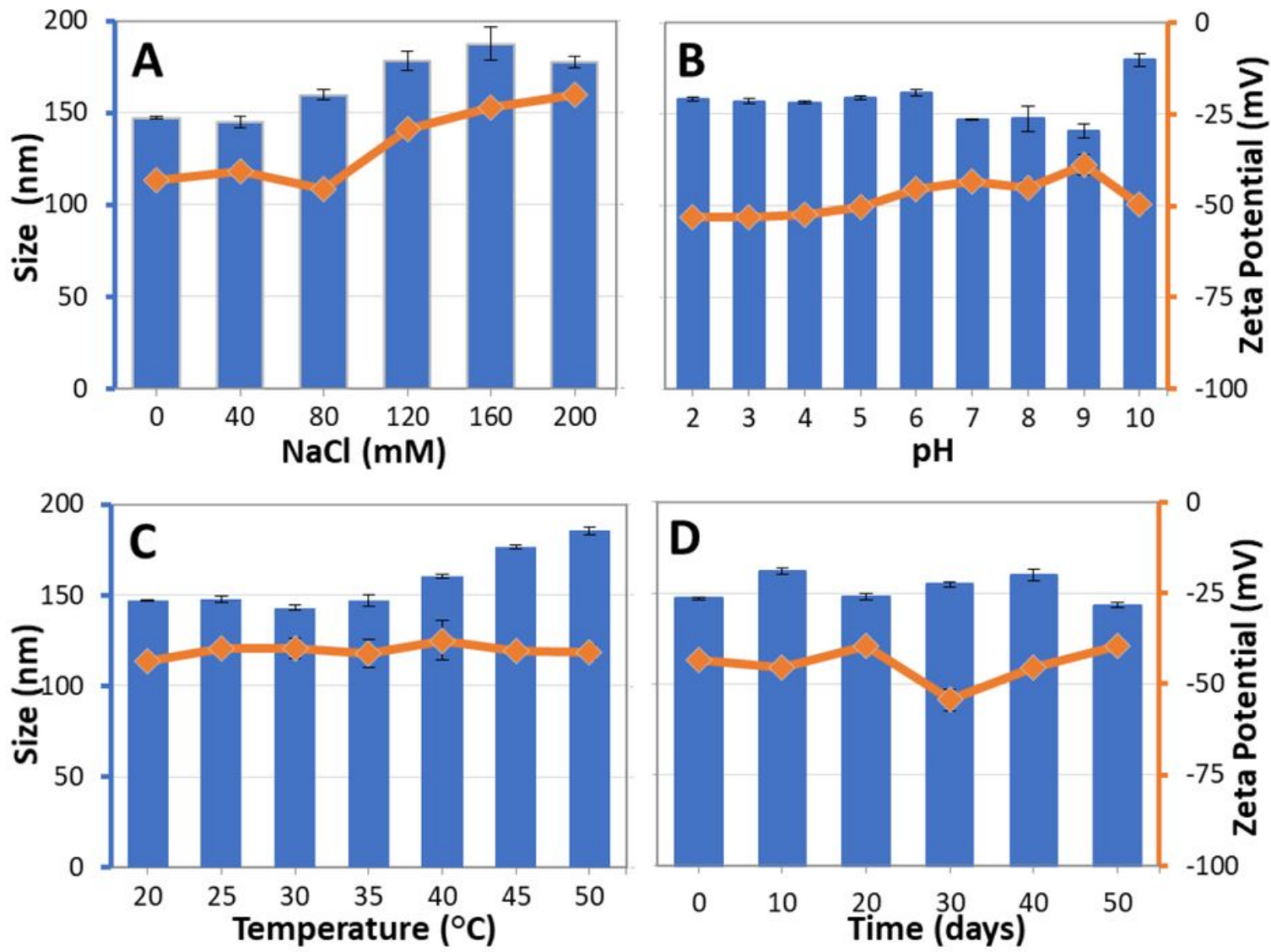
Figure 5

Stability study of NPs CQ/PSS 0.4 through evaluation of size (bars) and zeta potential (rhombuses), as a function of different $\mathrm{NaCl}$ concentrations (A), $\mathrm{pH}(\mathrm{B})$, temperature (C), and storage time (D) (mean $\pm \mathrm{SD}$, $n=3)$.

\section{Figure 6}

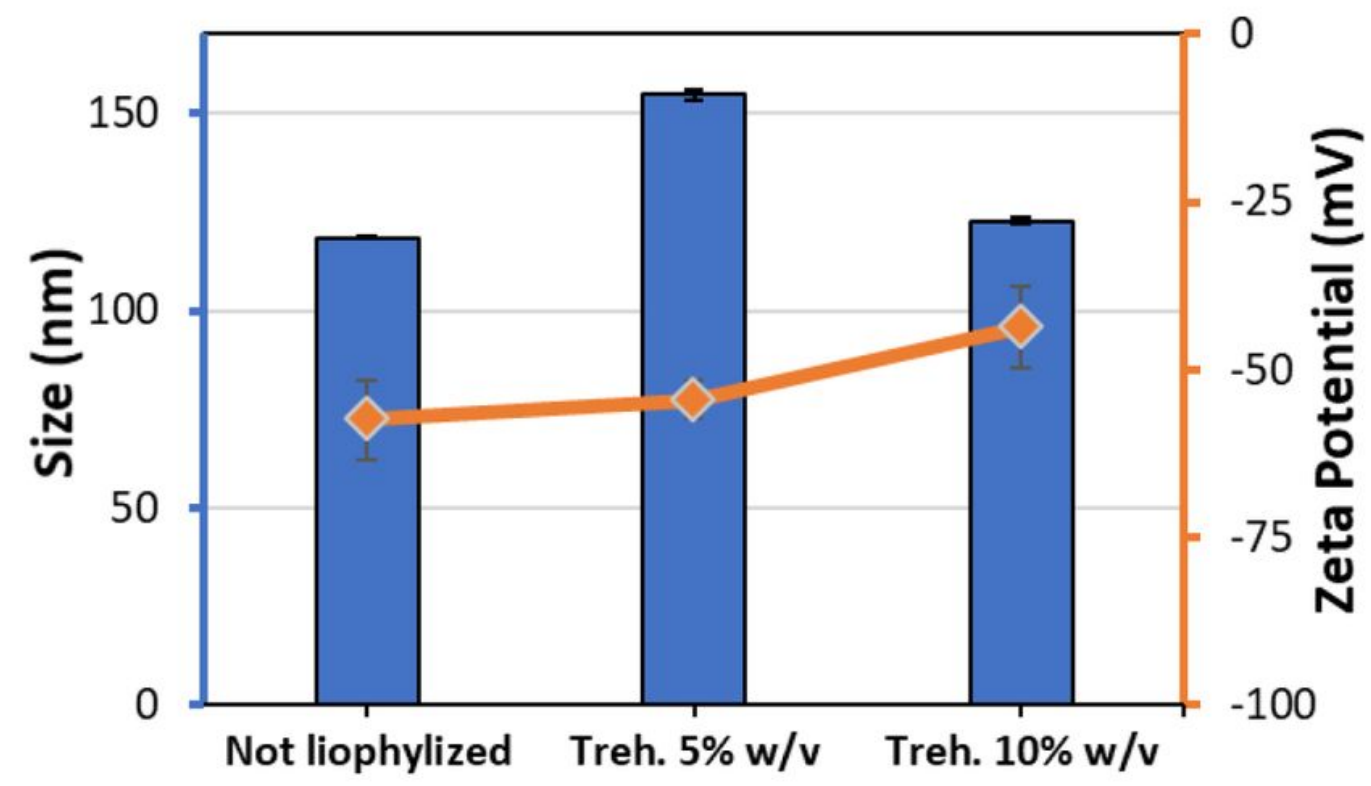

Figure 6

Size (bars) and zeta potential (rhombuses) variations for reconstituted freeze-dried NPs CQ/PSS 0.4 with different concentrations of trehalose ( $5 \%$ and $10 \%$ ) (mean $\pm S D, n=3$ ). 
Figure 7

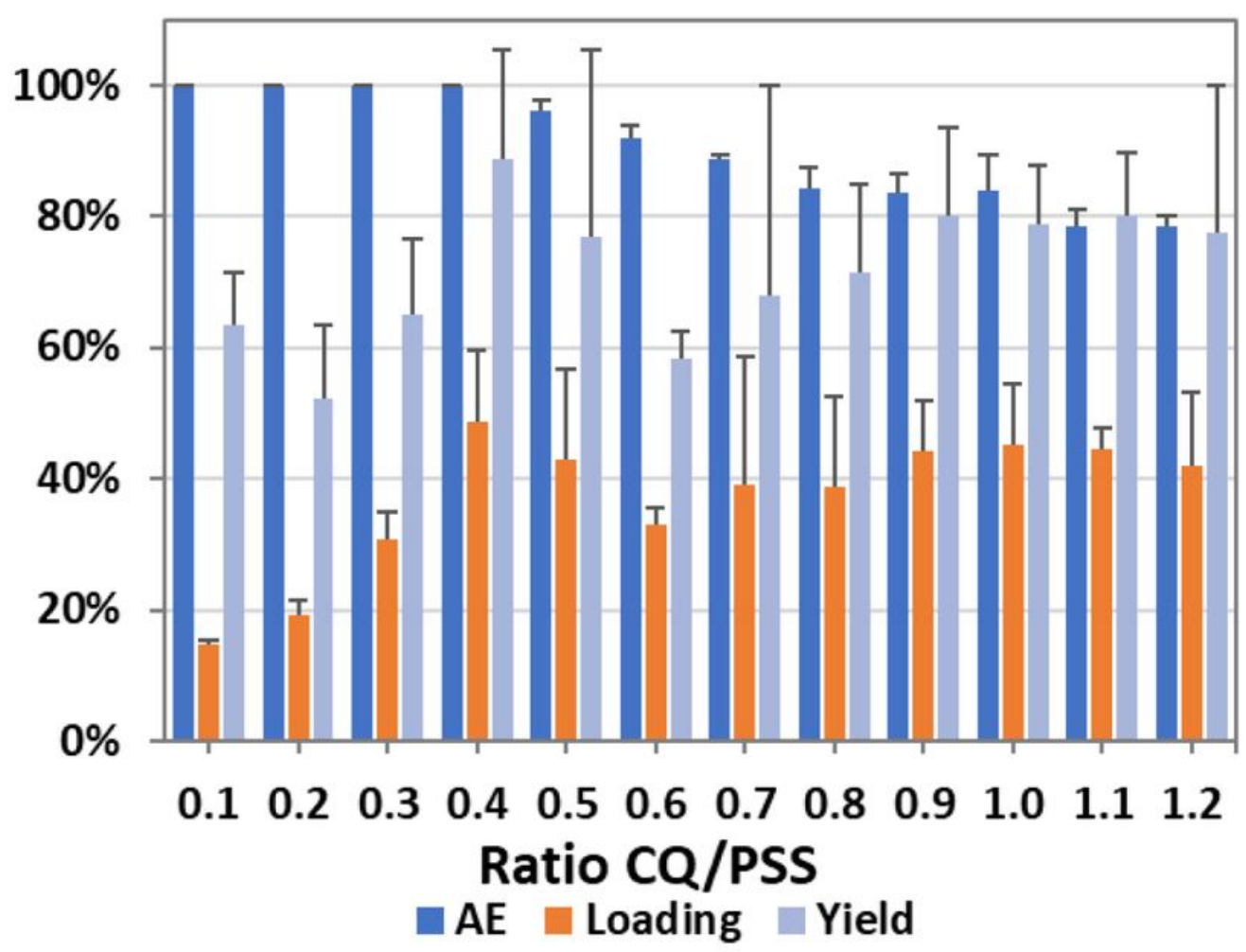

Figure 7

Association parameters of CQ/PSS formulations (mean $\pm S D, n=3$ ).

Figure 8
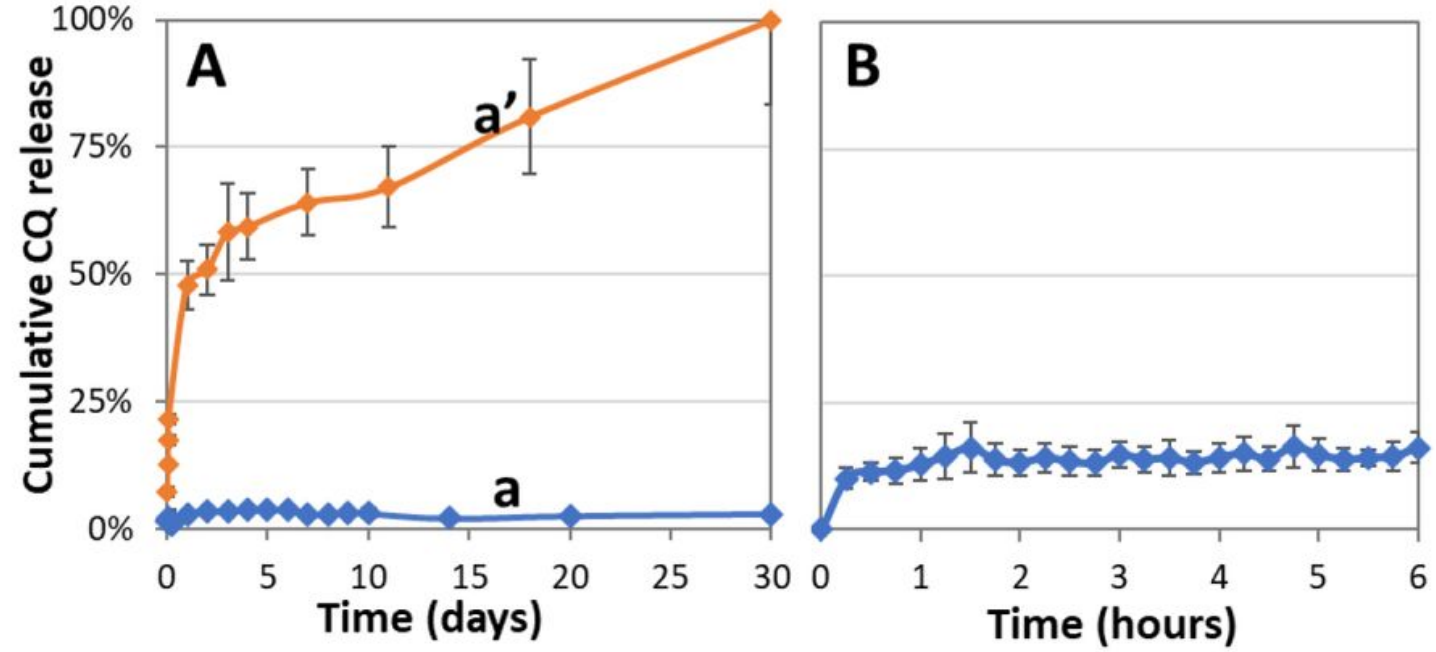


\section{Figure 8}

Cumulative release (\%) of CQ/PSS 0.4 NPs evaluated by conventional dialysis (A) in Milli-Q water (a) and simulated biological medium ( $\left.a^{\prime}\right)$, and by continuous flow cell USP 4 apparatus (B) (mean $\pm S D, n=3$ ).

\section{Supplementary Files}

This is a list of supplementary files associated with this preprint. Click to download.

- 201019SupplementaryInformation.docx 